\title{
THE POSSIBLE ORBITAL EVOLUTION OF THE NEAR-EARTH
}

\section{ASTEROIDS}

\author{
E.I.TIMOSHKOVA \\ Institute of Theoretical Astronomy, \\ Leningrad 191187, USSR
}

\begin{abstract}
The subject of this paper is a study of a possible orbital evolution for near-Earth asteroids. The investigation is fulfilled in the frame of the restricted circular three body problem. It is based on the calculations of the Jacobi constant. The osculating elements of some real ApolloAmor-Aten asteroids are used as the starting parameters. The comparison with the results of other authors is given.
\end{abstract}

\section{Introduction}

In the last few years there is large interest in the study of the near-Earth asteroids. The orbital evolution of these bodies has been considered by many authors (see, for example, Kozai, 1980; Vashkovjak, 1980). Here, the most important problem is the stability of the asteroid motions, because the understanding of many cosmogonical and geophysical problems has a connection with the orbital stability. It is known that large computer calculations are required in most of the methods of the evolution investigation.

The discoveries of new planet-crossing asteroids significantly increase the population of near-Earth asteroids every year and it is desirable to have some simple criterion in order to distinguish quickly the asteroids with the irregular behavior of orbits.

The Jacobi integral may be used as such criterion if the model of the circular restricted three-body problem is considered (Szebehely, 1979). It is known that the values of Jacobi constant $C$ for the stationary solutions of this problem are bifurcate and the behavior of the trajectories can be different for one and the same $C$. The 'measure of a motion stability' is defined as the derivation of the value of $C$ from the critical value $C\left(L_{i}\right)$ at the libration point. The first such approach has been used by $\mathrm{G}$.Hill in the investigation of the Moon orbital stability.

\section{Description of calculations}

In this paper the Jacobi constant was calculated for 135 near-Earth asteroids with perihelion distance up to $1.33 \mathrm{~A}$.U. Here we include 64 numbered asteroids from "Ephemerides of Minor Planets for 1991" and a large part of unnumbered asteroids. From these 135 objects we have 6 Aten , 70 Apollo and 59 Amor asteroids.

In the sidereal system of osculating elements the Jacobi constant will be defined by the Tisserand's criterion:

$$
J=C k^{-2}=\frac{1}{a}+\frac{2}{a_{j}} \sqrt{\frac{a\left(1-e^{2}\right)}{a_{j}(1-\mu)}}+\frac{\mu}{1-\mu} \delta
$$


and

$$
\delta=\frac{2}{\rho}+\frac{\rho^{2}-r^{2}-a_{j}^{2}}{a_{j}{ }^{3}}+\frac{\mu}{a_{j}}
$$

There $a, e, i$ are the semi-major axis, eccentricity and inclination of the asteroid, $a_{j}$ is the semi-major axis of the perturbing planet, $r$ is the Sun-asteroid distance, $\mu=m_{j} /\left(1+m_{j}\right), m_{j}$ is the mass of perturbing planet and $k$ is the Gauss constant. The solar mass is adopted for unit mass; the unit of distance is 1 A.U.

The formulae for the calculations of Jacobi constants $J\left(L_{i}\right)$ for five libration points $L_{i}$ are very well known and here we do not give them. The following disposition of libration points is used: $L_{3}$ is to the left of the Sun, $L_{1}$ is between the Sun and the perturbing planet and $L_{2}$ is to the right of planet.

The values of $J$ are calculated by formulae (1) and (2) for each asteroid, in the frame of the Sun-planet-asteroid problem, with the six perturbing planets from Mercury $(j=1)$ to Saturn $(j=6)$. The osculating orbital elements were taken as the initial data. For each perturbing planet, the values of $J\left(L_{i j}\right)$ are also determined. Then, we distinguish those asteroids for which $\left|J(N)-J\left(L_{i j}\right)\right|<10^{-1}$.

The results of other authors on the orbital evolution of some planet-crossing asteroids were analyzed for the comparison with our calculations. This is important to decide the question about the use of the difference $J(N)-J\left(L_{i}\right)$ for the prediction of unstable motions.

\section{Results}

Here we have no opportunity to produce all our results. Only some of these are summarized in Tables 1 and 2 . In the case of Saturn, the values of Jacobi constant for most of the asteroids differ very much from $J$ at the Saturnian libration points. So, we do not include this planet in Tables 1 and 2 .

From results given in Table 1 it follows that for some asteroids the Jacobi constant is very close to the corresponding value at the libration point at once for two or three planets. Apparently it means very unstable asteroid motion. Our conclusions closely correlate with the results of Vashkovjak (1980) and Steel and Baggaley (1985). We may expect very interesting trajectories for the asteroids from the lower part of Table 1. For example, the asteroid 1986 DA has the value of Jacobi constant near equal to the value of $J$ at the libration point $L_{1}$ for Jupiter. For a few asteroids, the value $J(N)$ is in the interval of values between the libration points $L_{2}, L_{3}$ or $L_{2}, L_{1}$ for Jupiter. It is interesting that four of these objects have a 5:2 mean motion resonance with Jupiter. The transitions to typical cometary orbits were discovered for the asteroids $1985 \mathrm{WA}$ and $1986 \mathrm{JK}$ by Hahn et al. (1989). They found that the asteroid 1986 DA shows increasing libration amplitude and the transition from the Alinda-class to the Oljato-class of the orbits. Also, the asteroid 1987 QB 'jumped' from $5: 2$ to $8: 3$ resonance.

In all cases it is the characteristic feature of the chaotic orbital evolution. The asteroid $1990 \mathrm{HA}$ shows the similarity of its parameters to the corresponding 1987 QB parameters, with the exception of the semi-major axis. Therefore, it is required 
TABIE I

The parameters of some selected Apollo-Amor asteroids

\begin{tabular}{|c|c|c|c|c|c|c|}
\hline $\mathrm{Nr}$ & Asteroid & $\bar{J}-\bar{J}\left(\bar{L}_{i j}\right)$ & $\bar{L}_{i j}$ & $a$ & $e$ & $i^{\circ}$ \\
\hline 433 & Eros & $\begin{array}{l}-0.001 \bar{\tau} \\
-0.0536\end{array}$ & $\begin{array}{l}\bar{L}_{43} \\
L_{44}\end{array}$ & 1.45832 & $0.2228 T$ & 10.826 \\
\hline 2202 & Pele & 0.00336 & $\overline{L_{13}}$ & 2.28941 & 0.51280 & 8.789 \\
\hline 3361 & Orplieus & $\begin{array}{c}-0.09363 \\
0.05853 \\
-0.03638\end{array}$ & $\begin{array}{l}L_{43} \\
L_{12} \\
L_{44}\end{array}$ & 1.20925 & 0.32265 & 2.688 \\
\hline 1943 & Anteros & $\begin{array}{l}-0.01519 \\
-0.05552\end{array}$ & $\begin{array}{l}L_{43} \\
L_{44}\end{array}$ & 1.43036 & 0.25589 & 8.704 \\
\hline 4015 & $1979 \mathrm{VA}$ & $\begin{array}{l}-0.08131 \\
-0.00844 \\
\end{array}$ & $\begin{array}{l}L_{43} \\
L_{15}\end{array}$ & 2.64109 & 0.62275 & 2.785 \\
\hline & $1982 \mathrm{DB}$ & $-0.0524 i$ & $L_{43}$ & 1.48954 & 0.36043 & 1.421 \\
\hline 3908 & $1980 \mathrm{PA}$ & -0.01546 & $L_{43}$ & 1.92504 & 0.45794 & 2.168 \\
\hline 1221 & Ámor & -0.0380 & $L_{43}$ & 1.91973 & 0.43535 & 11.898 \\
\hline 1620 & Geographos & -0.01896 & $L_{42}$ & 1.24473 & 0.33538 & 13.320 \\
\hline$\overline{2063}$ & Bacchus & $\begin{array}{r}-0.10002 \\
-0.02058 \\
\end{array}$ & $\begin{array}{l}L_{42} \\
L_{44}\end{array}$ & 1.07754 & 0.34940 & 9.419 \\
\hline & $1980 \mathrm{WF}$ & -0.00551 & $L_{43}$ & $2.2307 T$ & 0.51407 & 6.412 \\
\hline 3288 & Selencus & $\begin{array}{c}0.01340 \\
-0.00640 \\
0.00062\end{array}$ & $\begin{array}{l}L_{13} \\
L_{25} \\
L_{35} \\
\end{array}$ & $2.032 T 1$ & 0.45723 & 5.932 \\
\hline 1036 & Ganymed & $\begin{array}{c}-0.00073 \\
0.00628 \\
\end{array}$ & $\begin{array}{l}L_{25} \\
L_{35} \\
\end{array}$ & $2.6656 \mathrm{~T}$ & 0.53710 & 26.482 \\
\hline & $1985 \overline{\mathrm{WA}}$ & $\begin{array}{c}-0.00114 \\
0.00715\end{array}$ & $\begin{array}{l}L_{45} \\
L_{13}\end{array}$ & 2.84596 & 0.60143 & $9 . \overline{143}$ \\
\hline & $1986 \mathrm{JK}$ & -0.01283 & $L_{45}$ & $2.801 \overline{1} 3$ & 0.67972 & 2.140 \\
\hline & $1986 \mathrm{DA}$ & 0.00002 & $\overline{L_{15}}$ & 2.81129 & 0.58532 & 4.296 \\
\hline & $1987 \mathrm{QB}$ & $\begin{array}{c}-0.00050 \\
0.00652\end{array}$ & $\begin{array}{l}L_{25} \\
L_{35}\end{array}$ & 2.80255 & 0.59368 & 3.462 \\
\hline & $1983 \mathrm{TF} 2$ & $\begin{array}{c}-0.00158 \\
0.00543\end{array}$ & $\begin{array}{l}L_{25} \\
L_{35}\end{array}$ & 2.43893 & 0.73633 & $14 . \overline{104}$ \\
\hline & $1987 \mathrm{PA}$ & $\begin{array}{l}0.00451 \\
0.00397 \\
\end{array}$ & $\begin{array}{l}L_{13} \\
L_{15} \\
\end{array}$ & 2.73623 & 0.55681 & 16.121 \\
\hline & $1990 \mathrm{IIA}$ & $\begin{array}{c}-0.00004 \\
0.00020 \\
-0.00699\end{array}$ & $\begin{array}{l}L_{15} \\
L_{25} \\
L_{42}\end{array}$ & 2.56692 & 0.69212 & 3.882 \\
\hline
\end{tabular}


TABLE II

The values of Jacobi constant. $J$ for 6 Aten-asteroids

\begin{tabular}{|lr|c|c|c|c|c|c|}
\hline $\mathrm{Nr}$ & Asteroid & $q$ & Mercury & Venus & Earth & Mars & Jupiter \\
\hline 2340 & Hathor & 0.4643 & $\overline{T .9697}$ & 3.8384 & 2.8173 & 2.0529 & 1.3228 \\
2100 & Ra-Shalom & 0.4689 & $\underline{\tau .7608}$ & 3.7696 & 2.7815 & 2.0418 & 1.3353 \\
2062 & Aten & 0.7900 & 8.6269 & 4.0070 & 2.8632 & $\underline{2.0070}$ & $\underline{1.1892}$ \\
& $1954 \mathrm{XA}$ & 0.5088 & 8.1408 & 3.9701 & $\underline{2.9374}$ & 2.1644 & 1.4261 \\
& $1989 \mathrm{UQ}$ & 0.6711 & 8.7473 & $\underline{4.0895}$ & $\underline{2.9362}$ & 2.0730 & 1.2484 \\
& $1989 \mathrm{VA}$ & 0.2951 & 6.3643 & 3.3268 & 2.5748 & 2.0118 & 1.4742 \\
\hline$J\left(L_{1}\right)$ & & $\tau .7503$ & 4.1486 & 3.0009 & 1.9692 & 0.5846 \\
\hline$J\left(L_{4}\right)$ & & $\tau .7500$ & 4.1475 & 3.0000 & 1.9690 & 0.5769 \\
\hline
\end{tabular}

to study the long time orbit evolution of $1990 \mathrm{HA}$ by numerical integration.

From Table 2 it is seen that for all Aten-asteroids in problem with Mars, Jupiter and also Saturn (not shown in the table) we have $J(N)>J\left(L_{1}\right)$. It is true also for Mercury, except for asteroid $1989 \mathrm{VA}$. This result means that the movement of these asteroids is Hill-stable and they can not catch in the nearest neighborhood of the corresponding perturbing planet. On the contrary, in the cases of Earth and Venus we have $J(N)<J\left(L_{4}\right)$ and the Hill-stability of the asteroid orbits is absent.

The results presented here should be considered as preliminary. Nevertheless, it seems that the Jacobi constant can be used to distinguish the asteroids with possible irregular motion.

\section{References}

Hahn, G., Lagerkvist, C., Lindgren, M. and Dahlgren, M.:1989 in C.I.Lagerkvist et al., eds., Asteroids, Comets, Meteors III, Uppsala, p.95.

Kozai, Y.:1980, Icarus 41, 1.

Steel, D.I. and Baggaley, W.J.:1985, Mon. Not. R. Astr. Soc. 212 (4), 817.

Szebehely, V.:1979 in V. Szebehely, ed., Instabilities in Dynamical Systems Reidel, Dordrecht, p.115.

Vashkovjak, M.A.:1980, Inst. Appl. Math. USSR Academy of Sciences, Preprint No. 93.

Ephemerides of Minor Planets for 1991, Nauka, Institute of Theoretical Astronomy, Leningrad. 